\title{
Use of the SAW sensor electronic nose for detecting the adulteration of virgin coconut oil with RBD palm kernel olein.
}

\begin{abstract}
An electronic nose (zNose ${ }^{\mathrm{TM}}$ ) was applied to the detection of adulteration of virgin coconut oil. The system, which is based on a surface acoustic wave sensor was used to generate a pattern of volatile compounds present in the samples. Virgin coconut oil was mixed with refined, bleached and deodorized palm kernel olein at a level of adulteration from 1 to 20\% $(\mathrm{wt} / \mathrm{wt})$. Adulterant peaks were identified from the chromatogram profile and fitted to a curve using linear regression. The best relationship ( $2=0.91)$ was obtained between the peak tentatively identified as methyl dodecanoate and the percentage of palm kernel olein added. Pearson's correlation coefficients (r) of 0.92 and 0.89 were obtained between adulterant peak methyl dodecanoate and of the iodine and peroxide values, respectively. Principal component analysis (PCA) was used to differentiate between pure and adulterated samples. The PCA provided good differentiation of samples with $74 \%$ of the variation accounted for by PC 1 and $17 \%$ accounted for by PC 2. Pure samples formed a separate cluster from all of the adulterated samples.
\end{abstract}

Keyword: Adulteration; Electronic nose; Palm kernel olein; Principal component analysis Virgin coconut oil. 